\title{
Freno de banda
}

\section{Band brake}

\author{
Emilio A. Rivera-Landeros ${ }^{[a]}$, Martín Ortiz Domínguez ${ }^{[\mathrm{b}]}$, Arturo Cruz Avilés ${ }^{[\mathrm{cl}}$, Francisca \\ Santana Robles ${ }^{[\mathrm{dd}]}$, Yira Muñoz Sánchez ${ }^{[\mathrm{e}]}$, María E. Alcántara Hernández ${ }^{[\mathrm{fl}]}$, Leticia Villamil \\ Navarrete ${ }^{[\mathrm{g}]}$.
}

\section{Resumen}

El freno es un dispositivo usado para detener o disminuir la velocidad del consolador de algún cuerpo, generalmente, un eje, árbol o tambor. Los frenos son transformadores de energía, por lo que pueden ser entendidos como una máquina per se, ya que transforman la energía cinética de un cuerpo en calor o trabajo y en este sentido pueden visualizarse como "extractores" de energía. A pesar de que los frenos son también máquinas, generalmente se encuentran en la literatura del diseño como un elemento de máquina y en literaturas de teoría de control pueden encontrarse como actuadores. En el freno de banda, una banda de acero flexible forrada con material de fricción, presiona contra el tambor de freno giratorio. La acción de frenado se realiza para disminuir la velocidad o detener el tambor. La acción de frenado se obtiene apretando la banda alrededor del tambor. Este tipo de freno se utiliza en la máquina de urdido seccional y en el movimiento de distorsión de urdimbre en telares convencionales. Los frenos de banda se clasifican en frenos de banda simple y diferencial. En el presente estudio se propuso una deducción algebraica de la Presión Máxima ( $P_{\text {máx }}=$ $\left.2 P_{1} / b D\right)$.

Palabras clave: Presión máxima, Coeficiente de fricción, Torque, Ángulo de contacto 


\begin{abstract}
The brake is a device used to stop or slow down the comfort of a body, usually an axle, tree or drum. The brakes are energy transformers, so they can be understood as a machine, since they transform the kinetic energy of a body into heat or work and in this sense can be visualized as "extractors" of energy. Although brakes are also machines, they are generally found in the literature of design as a machine element and in literature of control theory they can be found as actuators. In band brake, a flexible steel band lined with friction material, presses against the rotating brake drum. The braking action is performed either to slow down or halting the drum. The braking action is obtained by tightening the band around the drum. This kind of brake is used on sectional warping machine and warp let-off motion on conventional looms. Band brakes are classified into simple and differential band brakes. In the present study an algebraic deduction of the Maximum Pressure was proposed $\left(P_{\text {máx }}=\right.$ $\left.2 P_{1} / b D\right)$.
\end{abstract}

Key words: Maximum pressure, Coefficient of friction, Torque, Contact angle

\title{
Introducción
}

Los frenos o embragues de tambor aplican un material de fricción a la circunferencia de un cilindro, ya sea externo o interno o de ambos lados. El freno de banda es la configuración más simple del freno de tambor, en el cual se enrolla una zapata flexible alrededor de la mayoría de la circunferencia exterior del tambor y se presiona contra él. Alternativamente; se puede pivotar una zapata forrada relativamente rígida, contra la circunferencia interior o exterior del tambor. El freno de banda flexible se emplea en excavadoras de potencia, en malacates y otros tipos de maquinaria (Norton, 2011).

El objetivo de este trabajo de investigación se centra en proporcionar una expresión matemática con la finalidad de ilustrar sus características para contrastarla básicamente con los frenos de disco. 


\section{Freno de banda}

Se analiza el movimiento rotatorio que tiene la función de almacenar y/o transferir energía cinética de rotación. Una representación dinámica simplificada de un freno de banda se muestra en la Figura 1. El freno se activa tirando fuertemente la banda contra el tambor de frenado. Se considera que la banda recubre uniformemente todo el ángulo de abrace $\theta$. Existen dos fuerzas activas sobre la banda $P_{1}$ y $P_{2}$, una activa de frenado y la otra reactiva en el soporte. Sin embrago, debido a la fricción existente se puede verificar la siguiente relación $P_{2}<P_{1}$ (Budynas et al., (2008).
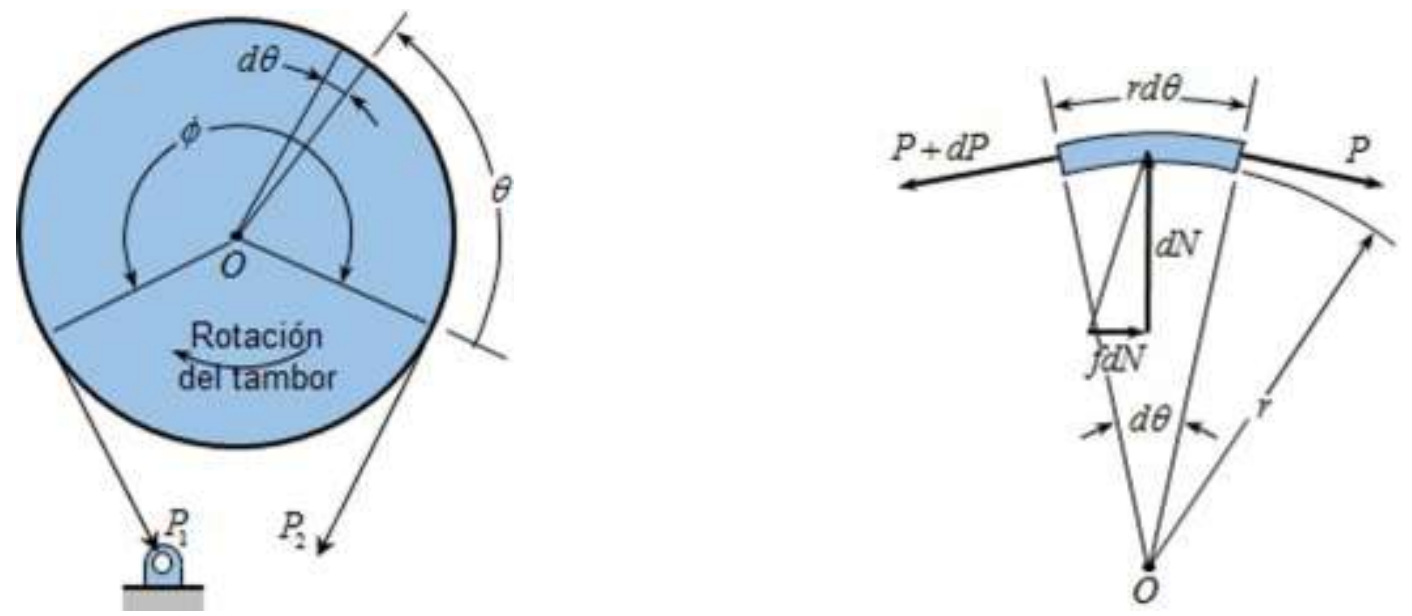

Figura 1. Fuerzas en un freno de banda.

(Budynas and Nisbett, 2008).

En la Figura 2. Se muestra un esquema para el análisis de los frenos de banda equilibrando fuerzas direccionales radial y circunferencial. 


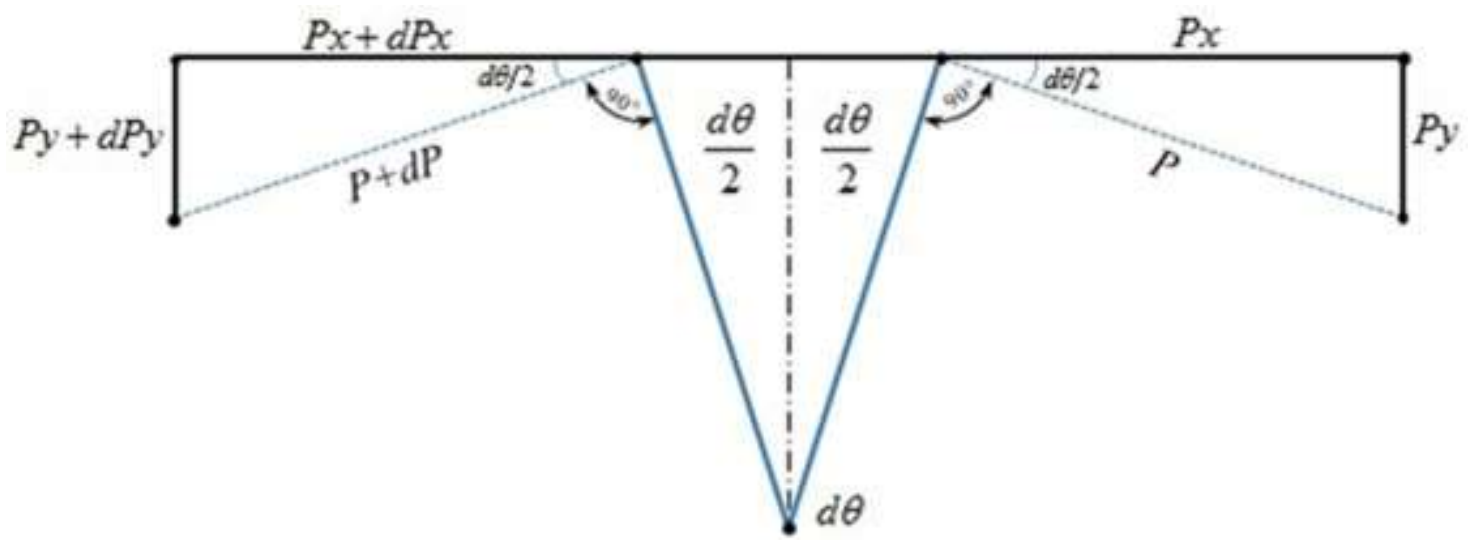

Figura 2. Esquema para análisis de frenos de zapatas accionados por banda.

(Elaboración propia)

Considerando la Figura 2, la mitad del lado izquierdo se tiene que:

$\sin \frac{d \theta}{2}=\frac{-(P y+d P y)}{P+d P}$

Despejando la ecu (1), tenemos;

$$
P y+d P y=-(P+d P) \sin \frac{d \theta}{2},
$$

También se tiene que

$$
\cos \frac{d \theta}{2}=\frac{-(P x+d P x)}{p+d P}
$$

Despejando la ecu. (3);

$$
P x+d P x=-(P+d P) \cos \frac{d \theta}{2},
$$

Ahora consideremos la Figura 2, la mitad del lado derecho se tiene: 
$\sin \frac{d \theta}{2}=\frac{-P y}{P}$

Despejando la Ec. (5):

$$
P y=-P \sin \frac{d \theta}{2}
$$

También se tiene:

$$
\cos \frac{d \theta}{2}=\frac{P x}{P}
$$

Despejando la Ec. (7) tenemos:

$$
P x=-P \cos \frac{d \theta}{2}
$$

Desarrollando una sumatoria de fuerzas en el eje $y$, se llega a:

$$
\begin{aligned}
& \sum F y=0, \\
& \sum F y=(P y+d P y)+P y+d N=0,
\end{aligned}
$$

Sustituyendo la Ec. (2) y Ec. (6) en Ec. (9), se obtiene:

$$
-(P+d P) \sin \frac{d \theta}{2}-P \sin \frac{d \theta}{2}+d N=0
$$

Por otro lado, para la sumatoria de fuerzas en el eje $x$ : 


$$
\begin{aligned}
& \sum F x=0, \\
& \sum F x=(P x+d P x)+P x+\mu d N=0,
\end{aligned}
$$

Sustituyendo la Ec. (4) y Ec. (8) en Ec. (11), se obtiene:

$$
-(P+d P) \cos \frac{d \theta}{2}+P \cos \frac{d \theta}{2}+\mu d N=0,
$$

Desarrollando las Ec. (10) y Ec. (12) y multiplicando por -1 en ambos lados se obtiene:

$$
\begin{aligned}
& P \sin \frac{d \theta}{2}+d P \sin \frac{d \theta}{2}+P \sin \frac{d \theta}{2}-d N=0, \\
& P \cos \frac{d \theta}{2}+d P \cos \frac{d \theta}{2}-P \cos \frac{d \theta}{2}-\mu d N=0,
\end{aligned}
$$

Aproximando la Ec. (13) por serie de Taylor $\sin \frac{d \theta}{2}=\frac{d \theta}{2}$, se tiene:

$$
P \frac{d \theta}{2}+d P \frac{d \theta}{2}+P \frac{d \theta}{2}-d N=0
$$

Sabiendo que el producto de los diferenciales es $\approx 0$,

$$
2 P \frac{d \theta}{2}-d N=0
$$

Entonces la Ec. 16 la podemos expresar como:

$$
d N=P d \theta
$$

Aproximando la Ec. (4) por serie de Taylor $\left(\cos \frac{d \theta}{2} \approx 1\right)$, se obtiene: 


$$
P+d P-P-\mu d N=0,
$$

Simplificando la Ec. (18), se obtiene:

$$
d P=\mu d N
$$

Sustituyendo la Ec. (17) en la Ec. (19), tenemos:

$$
\mu P d \theta=d P
$$

Despejando la Ec. (20), se tiene:

$$
\frac{d P}{P}=\mu d \theta
$$

Integrando la Ec. (21),

$$
\begin{aligned}
& \int_{P_{2}}^{P_{1}} \frac{d P}{P}=\mu \int_{0}^{\emptyset} d \theta, \\
& \ln \frac{P_{1}}{P_{2}}=\mu \emptyset,
\end{aligned}
$$

Despejando $\frac{P_{1}}{P_{2}}$ ( $P_{1}$ fuerza de tensión de la banda y $P_{2}$ fuerza floja de la banda) de la Ec. (23), se obtiene:

$$
\begin{aligned}
& e^{\ln \left(\frac{P_{1}}{P_{2}}\right)}=e^{\mu \emptyset}, \\
& \frac{P_{1}}{P_{2}}=e^{\mu \emptyset} .
\end{aligned}
$$


Se le conoce al par de torsión como un sistema formado por dos fuerzas de la misma intensidad o módulo, pero en sentido contrario. Al aplicar un par de fuerzas a un cuerpo se produce una rotación o una torsión. El par de torsión del sistema de freno de banda puede obtenerse de las ecuaciones:

$$
\begin{aligned}
& T_{1}=P_{2} \frac{D}{2}, \\
& T_{2}=P_{2} \frac{D}{2},
\end{aligned}
$$

El Par de torsión total se obtiene de,

$$
T_{t}=T_{1}-T_{2}
$$

Sustituyendo la ec. (26) y Ec. (27) en la Ec. (28), se obtiene:

$$
T_{t}=P_{1} \frac{D}{2}-P_{2} \frac{D}{2}
$$

Simplificando la Ec. (29), tenemos la capacidad de frenado o par torsional neto sobre el tambor:

$$
T_{t}\left(P_{1}-P_{2}\right) \frac{D}{2}
$$

La fuerza normal $d N$ que actúa sobre un elemento de área de anchura $b$ y longitud $r d \theta$ es:

$$
p=\frac{d F}{d A}=\frac{d N}{d A^{\prime}},
$$

Donde $d A^{\prime}$ es: 


$$
d A^{\prime}=b(r d \theta)
$$

Sustituyendo la Ec. (32) en la Ec. (31), se obtiene:

$$
p=\frac{d N}{b(r d \theta)},
$$

Despejando la Ec. (33), se tiene:

$$
d N=p b(r d \theta),
$$

Sustituyendo la Ec. (17) en la Ec. (34), se obtiene:

$$
P d \theta=p b(r d \theta),
$$

Simplificando la Ec. (35), se reescribe como:

$$
P=p b r,
$$

Finalmente se obtiene:

$$
p=\frac{P}{b r}=\frac{2 P}{d D},
$$

Por lo tanto se tiene que la presión máxima aplicada en el freno de banda la podemos calcular con la siguiente Ecuación.

$$
p_{\text {máx }}=\frac{2 P}{b D} .
$$




\section{Resultados y discusiones}

Resulta útil contar con una expresión matemática (ver Ec. (34)) que permite obtener la presión máxima aplicada a un sistema de freno por banda. Asimismo, es importante tener en cuenta que varía en consecuencia con la variación de la presión en la banda, desde el máximo valor en la rama tensa, hasta el mínimo valor en la rama floja.

\section{Conclusiones}

El propósito de este trabajo fue proponer una deducción algebraica de la Presión Máxima $\left(P_{\text {máx }}=2 P_{1} / b D\right)$ en un freno de banda, a partir de la fuerza de tensión en la banda o tensión máxima en la banda $\left(P_{1}\right)$, la fuerza floja en la banda o tensión mínima en la banda $\left(P_{2}\right)$, el ancho de banda $(b)$, el diámetro del tambor $(D)$, ángulo de contacto entre la banda y el tambor $(\emptyset)$ y el coeficiente de fricción $(\mu)$. Asimismo, se demostró la capacidad de frenado o par torsional neto sobre el tambor $(T)$ empleado en excavadoras de potencia y en malacates y otros tipos de maquinaria.

\section{Agradecimientos}

El trabajo descrito en el presente artículo fue financiado por la beca otorgada por PRODEP. Asimismo, los autores le agradecen al Dr. Jorge Zuno Silva, quien es Director de la Escuela Superior de Ciudad Sahagún-UAEH, México, por todas las facilidades para concluir este trabajo de investigación. 


\section{Referencias}

Budynas, R. G., and Nisbett, J. K. (2008). Diseño en ingeniería mecánica de Shigley. D.F., México: MacGraw-Hill.

Norton, R. L. (2011). Diseño de Máquinas. Estado de México, México: Prentice Hall Hispanoamérica, S. A.

Shigley, J. E., and Mischke, C. R. (1989). Mechanical Engineering Design. New York, US: MacGraw-Hill.

Madayag, A. F. (1969). Matal Fatigue: Theory and Design. New York, US: John Wiley \& Sons

Dowling, N. E. (1993). Mechanical Behavior of Materials. New York-Englewood Cliffs, US: Prentice Hall.

Norton, R. L. (1999). Design of Machinery: An Introduction tho the Synthesis and Analysis of Mechanisms and Mechines. New York, US: McGraw-Hill.

Proyectos de elementos de acoplamiento, Recuperado 17 de julio de 2017, de https://www.frbb.utn.edu.ar/frbb/images/carreras/elementosdemaquinas/cap0801.pdf 
[a]Licenciatura en Contaduría, Escuela Superior de Ciudad Sahagún, UAEH, Tepeapulco, Hidalgo, México, rlandero@uaeh.edu.mx;

${ }^{[b]}$ Licenciatura en Ingeniería Mecánica, Escuela Superior de Ciudad Sahagún, UAEH, Tepeapulco, Hidalgo, México, martin ortiz@uaeh.edu.mx, 0000-0003-4475-9804;

[c] Licenciatura en Ingeniería Mecánica, Escuela Superior de Ciudad Sahagún, UAEH, Tepeapulco, Hidalgo, México, arturo_cruz8085@uaeh.edu.mx, 0000-0003-0455-1646;

[d] Licenciatura en Ingeniería Industrial, Escuela Superior de Ciudad Sahagún, UAEH, Tepeapulco, Hidalgo, México, profe_7739@uaeh.edu.mx, 0000-0002-3301-9790;

[e] Licenciatura en Ingeniería Mecánica, Escuela Superior de Ciudad Sahagún, UAEH, Tepeapulco, Hidalgo, México, yira@uaeh.edu.mx;

${ }^{[1}$ Licenciatura en Ingeniería Industrial, Escuela Superior de Ciudad Sahagún, UAEH, Tepeapulco, Hidalgo, México, eugenia31702@yahoo.com.mx;

[q] Licenciatura en Contaduría, Escuela Superior de Ciudad Sahagún, UAEH, Tepeapulco, Hidalgo, México, letyvina@gmail.com; 